\title{
CONNECTING SUPPLY AND DEMAND VERTICES WITH FAULT TOLERANCE
}

\author{
Wen-Li Wang ${ }^{1}$, Chris Coulston ${ }^{2}$, and Robert Weissbach ${ }^{3}$, Mei-Huei Tang ${ }^{4}$ \\ ${ }^{123}$ School of Engineering, Penn State Erie, The Behrend College, Erie PA, USA

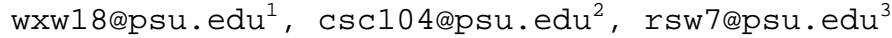 \\ ${ }^{4}$ Computer and Information Science Dept., Gannon University, Erie PA, USA \\ tang002@gannon.edu
}

\begin{abstract}
Fault tolerance can improve reliability and robustness when providing connectivity among entities. Many studies had accounted for entities that were uniform and similar in nature. However, applications today often require the entities to have distinguished roles. For instance, a power system has two types of entities: sources and loads. A source mainly concerns no disconnection from the network, while a load further favors connections to multiple sources to gain dependable services. Such non-uniformity in the requirements calls for a new fault tolerance modeling approach. This paper introduces a 2-edge supply/demand (2-ESD) connected problem. It is NP-hard like many connectivity problems. Algorithms are developed to satisfy different fault tolerance requirements of the entities. Edge reduction and refactoring are further employed to identify a better cost effective solution. Experiments were conducted to compare the cost of the derived 2-ESD network with that of the minimum spanning tree (MST) solution.
\end{abstract}

\section{KEYWORDS}

Fault tolerance, Algorithms, Graph Algorithms, NP-Hardness

\section{INTRODUCTION}

Fault tolerance should be an option when providing connectivity for entities with a high reliability demand. For example, in the electrical power grid, most substations (power loads) are connected by two or more transmission lines, ensuring that loss of one line does not result in loss of power to a substation $[1,2]$. The new renewable energy sources at remote locations, such as wind turbine farms, are expected to be integrated into the transmission system in the coming decades. Because the cost of installing transmission lines is estimated at $\$ 1.5 \mathrm{M} / \mathrm{mile}$ [3], there is a strong motivation to minimize the extent of this new infrastructure without compromising the network robustness.

The area of fault tolerant routing had been extensively studied. In [4], the authors provide a routine for routing of bi-connected graphs that is optimal for both vertex faults and edge faults. In [5], an optimal routing algorithm is presented for double loop communication networks with at most one faulty edge. The authors in [6] investigate fault tolerance of wireless networks while ensuring minimum power consumption for critical military and disaster relief applications. Similarly, the problem of connecting new power sources and loads to the electrical power grid can be depicted by a graph with vertices (e.g., wind farms and substations) and edges (e.g., transmission lines). 
Nevertheless, the prior work in fault tolerant routing only had to model uniform vertices with same connectivity demands. This is not applicable to the power grid problem in which the vertices can have different connectivity requirements. In reality, fault tolerance is more vital to the substations than the wind farms, because a power source going offline carries fewer consequences than a power load would do. A nonfunctional wind farm may likely be compensated by the other connected power sources, but a disconnected substation will result in power loss to a community. This real-world problem motivates our study to meet this new connectivity requirement for distinguished entities.

For the two types of entities, the vertices in the graph can be partitioned into two classes: supply/producer vertices, and demand/consumer vertices. This classification terminology has been used in other areas such as the supply chain [7] or transportation network [8]. The objective of this work is to ensure that fault tolerance is provided to all demand vertices without accounting for the supply vertices, because loss of a supply vertex is not deemed critical. Consequently, an edge disconnection to a demand vertex must not result in its loss of services from any other supply vertices. From the power point of view, one single edge failure will not cause a substation to disconnect with to all power sources. This problem is referred to as a 2-edge supply/demand (2-ESD) connected problem and its solution, a 2-ESD redundant graph. In our proposed algorithms, optimization is also employed to the solution to reduce the connection cost.

The rest of this paper is organized as follows. First, the 2-ESD problem is presented and some elementary properties are discussed to illustrate the problem's NP-hardness. Next a series of heuristic algorithms are presented to solve the 2-ESD problem. Finally, the experimental results compare the developed 2-ESD redundant graph with the MST network on the Euclidean distance and computational time.

\section{Properties}

The minimal cost 2-ESD connected problem is defined as follows. Given a complete graph $G=$ $(V, E)$, a partition of $V$ into two sets $S$ (for supply vertices) and $D$ (for demand vertices), and a cost function $C: E \rightarrow \mathbb{R}^{+}$, determine a least cost collection of edges $E^{\prime} \subseteq E$ connecting vertices in $S$ and $D$ into a single connected component with the property that the removal of any single edge in $E^{\prime}$ leaves at least one path from any vertex in $D$ to some vertex in $S$.

Menger's Theorem, a special case of the Max-flow Min-cut Theorem [9], can be used to provide a different perspective on 2-ESD connectivity. It states that the smallest size of an edge-cut which disconnects a graph is equal to the largest number of edge disjoint paths between any pair of vertices in the two components. In a 2-ESD connected graph, it would take the removal of at least two edges (an edge-cut of size 2) to disconnect a demand vertex from a supply vertex. Hence, a 2-ESD connected graph has, by Menger's Theorem, two edge-disjoint paths between a demand vertex and one or more supply vertices.

A closely related problem to the 2-ESD connectivity problem is the minimal cost 2-edge connected graph problem [10], defined as follows. Given a complete graph $G=(V, E)$ and a cost function $C: E \rightarrow \mathbb{R}^{+}$, determine a least cost collection of edges connecting the graph in a single connected component with the property that the removal of any single edge leaves the graph connected. This problem has long been known to be NP-complete [11].

The difference between the two problems is the differentiation of the vertices into supply and demand vertices which can lead to different solutions as shown in Figure 1. Although the two problems are not identical, the 2-edge connected problem can be used to explain that the 2-ESD connected problem is NP-hard by showing that an instance of the 2-edge connectivity problem 
can be transformed and solved as a 2-ESD instance.

\section{2-edge connected}

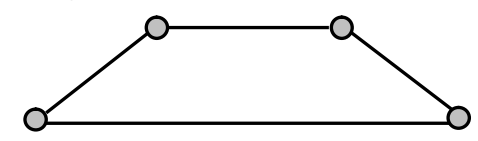

2-ESD connected

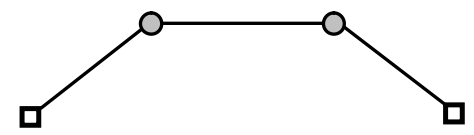

Figure 1. A 2-edge connected (left) network and 2-ESD connected network (right) on the same set of points. Supply vertices are shown as squares and demand vertices as circles.

Consider an instance of the minimal cost 2-edge connected graph problem, $I$, consisting of a graph $G=(V, E)$ and a cost function $C$. $I$ is transformed into an instance of the 2-ESD connected graph problem $I^{\prime}$ consisting of a graph $G^{\prime}=\left(V^{\prime}, E\right)$, a partition of the vertices $V^{\prime}=S \cup D$, and a cost function $C^{\prime}$. To accomplish this, first, set $V^{\prime}=V$ and place all but one vertex from $V$ into the set of demand vertices, $D$. Add the single remaining vertex, denoted $v^{\prime}$, to the set of supply vertices, $S$. Add all the edges from $E$ into $E^{\prime}$ and use the same cost function for each edge. Lemma 1 shows that the choice of which vertex to put into $S$ does not affect the cost of the solution.

\section{Lemma 1: In the construction of $I$, the choice of which vertex to select as $v^{\prime}$ does not change the cost of the solution to $I^{\prime}$.}

Proof: Assume to the contrary, that the cost of a solution to $I_{1}$, when vertex $v_{1}$ is put into $S$, is $c_{1}$. Also assume that the cost of a solution to $I_{2}$, when vertex $v_{2}$ is placed into $S$, is $c_{2}$ and that $c_{1}<c_{2}$. It can be shown that every vertex in $I_{1}$ has two edge-disjoint paths to $v_{2}$. Hence $I_{1}$ is a solution for $I_{2}$, contradicting the supposed minimality of $I_{2}$.

Since every vertex in $I_{1}$ is connected to $v_{1}$ by two edge-disjoint paths, then $v_{2}$ is connected to $v_{1}$ by two edge-disjoint paths. Any other vertex in $I_{1}$, denoted $v_{3}$, is connected to $v_{1}$ by two disjoint paths which interact with the paths of $v_{2}$ in a variety of ways, all of which are enumerated in Figure 2. In order to reduce the number of subcases, Figure 2 only shows the initial subpath of $v_{3}$ to $v_{1}$ which is independent of the paths from $v_{2}$ to $v_{1}$. In all cases there are two edge-disjoint paths from $v_{3}$ to $v_{2}$.

Since $v_{3}$ is any vertex in the graph, there are two edge-disjoint paths between every vertex and $v_{2}$. Since we assumed that solution $I_{1}$ had a lower cost, the solution for $I_{1}$ can be used as a lower cost solution for the instance $I_{2}$, contradicting the supposed minimality of $I_{2}$. Hence, the choice of vertex to select makes no difference to the cost of the resulting solution.

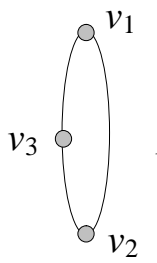

(a)

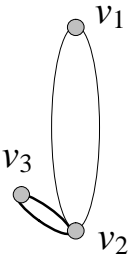

(b)

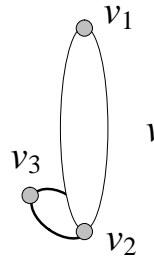

(c)

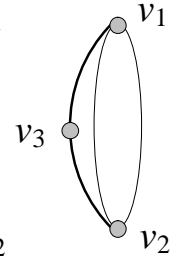

(d)

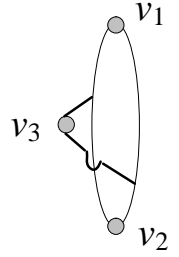

(e)

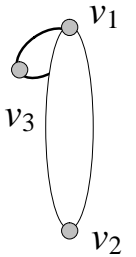

(f)

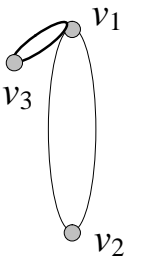

(g)

Figure 2. If every vertex in a 2-ESD connected graph has two edge-disjoint paths to supply vertex $v_{1}$, there are also two edge-disjoint paths to $v_{2}$.

As a corollary to Lemma 1, the network of edges created by the solution of the 2-ESD instance $I^{\prime}$ 
is 2-edge connected because every vertex has two edge-disjoint paths to $v_{2}$, an arbitrary vertex in the graph.

Theorem 1: Given a 2-edge connected instance $I$, create a 2-ESD instance $I^{\prime}$ by placing all but one vertex in the demand set and the remaining vertex in the supply set. An optimal solution to $I^{\prime}$ is an optimal solution to $I$.

Proof: Select an arbitrary vertex as $v^{\prime}$ to be the supply vertex in the 2-ESD instance $I^{\prime}$ and determine the optimal solution. Lemma 1 implies that there are two edge-disjoint paths between any pair of vertices in $I^{\prime}$, hence is 2 -edge connected. Thus, the optimal solution to $I$ cannot cost more than the optimal solution $I^{\prime}$, otherwise $I^{\prime}$ could be used as a lower cost solution.

Since the solution $I$ is 2-edge connected it could be used as a solution for $I^{\prime}$. Thus, the cost for the solution $I^{\prime}$ cannot be higher than the cost of the solution for $I$.

Since the solutions $I$ and $I^{\prime}$ cannot cost more than one another, they must have the same cost. Furthermore, since $I^{\prime}$ has a feasible structure for a solution to $I$, an optimal solution to the 2-ESD instance $I^{\prime}$, is an optimal solution to the 2-edge instance $I$.

With the solutions of $I$ and $I^{\prime}$ having the same cost, the problem of finding a 2-ESD connected network is at least as difficult as finding the 2-edge connected network. Consequently, the 2-ESD connected network problem is NP-Hard.

\section{ALgORITHM}

The heuristic algorithms employed to generate a solution to the 2-ESD problem can be separated into two main components, edge generation and edge reduction/refactoring. The routines developed to assist the edge-generation phase are explained in greater detail, because any heuristic algorithm which constructs a 2-ESD network incrementally will require similar routines. First, some definitions and properties are presented.

\subsection{Definitions and Properties}

A path is a sequence of vertices such that there is an edge between consecutive vertices in the sequence. A simple cycle is a path with no repeated edges and vertices other than the starting and ending vertices. A vertex is robust if it is a demand vertex that has two or more edge-disjoint paths to a supply vertex, or is a supply vertex. The degree of a vertex $v$, denoted as $\operatorname{deg}(v)$, is the number of incident edges of $v$. A demand vertex with degree one is called a singleton. When the degree of a vertex is greater than or equal to a value, it is described with a number followed by a "+". The cost of an edge is the Euclidean distance between the end points. The cost of a network is the sum of the cost of all of its edges.

Lemma 2: A demand vertex $v$ on a path $P$ which begins and ends on robust vertices $v_{r 1}$ and $v_{r 2}$ is also robust.

Proof: If $v_{r 1}$ and $v_{r 2}$ are the same vertex, denoted $v_{r}$, then $v$ lies on a simple cycle. The vertex $v$ has two edge-disjoint paths to $v_{r}$, one traversing the cycle clock-wise and the other counter-clockwise. The robust vertex $v_{r}$ has two edge disjoint paths to supply vertices $s_{1}$ and $s_{2}$ ( $s_{1}$ may be the same vertex as $s_{2}$ ). It is a simple matter to construct two edge-disjoint paths from $v$ to $s_{1}$ and $s_{2}$, via $v_{r}$, using these four edge-disjoint paths. 
Otherwise, $v_{r 1}$ and $v_{r 2}$ are distinct terminals of a path. In this case, the vertex $v$ has at least one path to a supply vertex formed by traversing the path from $v$ to $v_{r 1}$ and then onwards to one of $v_{r 1}$ supply vertices. Assume that $v$ is not robust then $v$ has only one path to a supply vertex through, without loss of generality, $v_{r 1}$. Since there are no edge disjoint paths from $v$ to a source through $v_{r 2}$, then both of the paths from the robust vertex $v_{r 2}$ to its associated supply vertices must share a common edge with the path from $v_{r 1}$ to its supply vertices. This implies that $v_{r 2}$ is not robust, a contradiction, consequently $v$ is robust.

The 2-ESD network is formed on the set of vertices in the Euclidian plane along with the corresponding edges between the vertices. The cost of an edge is the Euclidean distance between its associated vertices. Since the algorithm spends a significant amount of time processing edges, there is a strong motivation to reduce the size of the pool of $\mathrm{O}\left(|V|^{2}\right)$ candidate edges.

Call an edge, both of whose terminals are demand vertices, a demand-edge. It can be shown that in an optimal 2-ESD connected network, demand-edges do not cross. Hence, when connecting demand vertices, the algorithm only selects non-intersecting edges. To facilitate this selection, the algorithm draws edges from those in the Delaunay triangulation $[12,13]$.

A triangulation of a graph $G=(V, E)$ is a partitioning of the plane into triangles whose vertices are the vertices of the graph. A Delaunay triangulation consists of the vertices of $V$ along with a subset of edges $E^{\prime} \subseteq E$ which meet the empty-circle condition [12]. An edge $e$ is said to meet the empty-circle condition if there exists a circle circumscribing the end vertices that does not contain any other vertices of the graph. Since the edges of the Delaunay triangulation do not intersect one another, they form a convenient set from which to draw candidate edges of the 2-ESD network. It remains an open question whether an optimal 2-ESD connected network is constructed solely from edges in the Delaunay triangulation. Consequently, restricting an algorithm to this set of edges, while convenient for the formation of a redundant network, may result in a suboptimal solution.

\subsection{Generation of Redundant Edges}

The algorithm developed to solve the 2-ESD redundant problem starts by forming a minimum spanning tree (MST) over all the points in the graph making no distinction between the supply and demand vertices. Figure 3 shows the MST (in bold lines) over an instance with 4 supply vertices (squares) and 11 demand vertices (circles). The supply and demand vertices are labeled with an "s" and " $d$ ", respectively, and subscripted with an index. The edges of the Delaunay triangulation are the union of the dotted lines and the MST edges. There are five singletons $d_{1}$, $d_{2}, d_{4}, d_{5}$ and $d_{10}$.

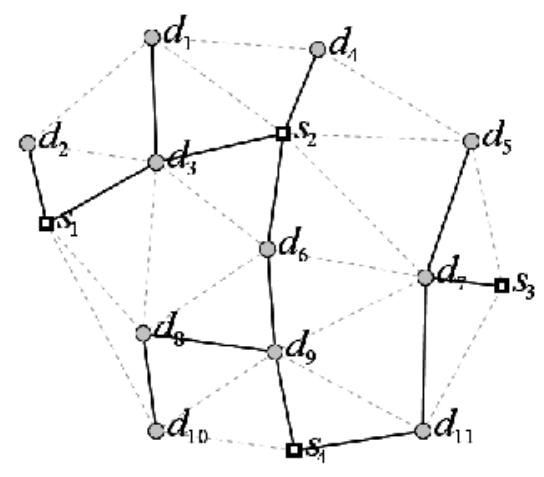

Figure 3. The MST, built from Delaunay triangulation, for the set of 11 demand vertices and 4 supply vertices. 
Using the MST as a starting point edges are added iteratively to singletons with the aim of converting them into robust vertices. To do this the algorithm keeps track of the vertices that are robust by putting them into a set $\boldsymbol{R}$. With respect to Figure $3, \boldsymbol{R}=\left\{d_{3}, d_{6}, d_{7}, d_{9}, d_{11}, s_{1}, s_{2}, s_{3}, s_{4}\right\}$. Since the MST connects all the vertices together, every non-robust demand vertex has at least one path to a supply vertex. Thus, by Lemma 2 , all that is required to convert a singleton into a robust vertex is to add an edge to connect it to a robust vertex. For example, adding the edge $\left\langle d_{10}, s_{4}\right\rangle$ to the graph in Figure 3 makes vertices $d_{10}$ and $d_{8}$ robust and consequently new elements of $\boldsymbol{R}$. Thus, the problem of building a 2-ESD network is reduced to finding the best edge to add to the singletons. To do this, a greedy approach is used, selecting the edge with the least cost which increases the cardinality of $\boldsymbol{R}$.

The getRobustSet routine in Listing 2 assists in the process of selecting an edge by returning a set of vertices in the graph which are robust. The routine explores the graph in a depth first manner starting at an arbitrary supply vertex to form a set of independent paths stored in a 2-dimensional array $\boldsymbol{A}$ using the recursive depthFirst routine in Listing 1.

Prior to calling the depthFirst routine the caller must initialize all the edges as unvisited, the $i d x$ and tail globals to 0 , and $\boldsymbol{A}[0][0]=v$, where $v$ is the vertex which the depthFirst routine is called with. Independent paths are stored in rows of $\boldsymbol{A}$ as sequences of vertices. Each row of $\boldsymbol{A}$ corresponds to an independent path stored in a compressed format, e.g., a path consisting of three edges $\langle a, b\rangle,\langle b, c\rangle$, and $\langle c, d\rangle$ is stored as ' $a, b, c, d$ ' in a row of $\boldsymbol{A}$. Routine depthFirst is called from the terminal vertex of the currently traversed edge on the path. At this vertex depthFirst chooses an untraversed edge from the vertex (line 2), marks this edge as traversed (line 3 ), and adds the edge to the current independent path. If there are no untraversed edges, execution returns to the caller. If a vertex has more than one untraversed edge, each edge after the first will create a new independent path. Consequently, the new_path flag is set (line 10) after the first iteration of the main for loop (line 2). Each subsequent iteration of the main for loop will result in the creation of a new independent path by incrementing the row index of the $\boldsymbol{A}$ array (line 6) and resetting the pointer (line 5). If this is the first edge of the independent path, both the starting and ending vertices are stored in $\boldsymbol{A}$ (line 6,7); afterwards just the terminal vertex of the edge is stored (line 9). After traversing an edge, the depthFirst routine is recursively called (line 11) at the new terminal vertex of the selected edge.

Listing 1 . Independent paths created by a depth first traversal of the spanning structure.

\begin{tabular}{|c|c|c|}
\hline \multicolumn{2}{|c|}{ depthFirst (v) } & // v vertex \\
\hline 1 & new_path = FALSE; & // local \\
\hline 2 & for all untraversed edges $e=\langle\mathrm{v}, \mathrm{w}\rangle$ & \\
\hline 3 & traversed $(e) \leftarrow$ TRUE; & \\
\hline 4 & if new_path & \\
\hline 5 & tail $\leftarrow 0$ & \\
\hline 6 & $A[++i d x][$ tail $] \leftarrow v ;$ & \\
\hline 7 & $A[i d x][++$ tail $] \leftarrow w ;$ & \\
\hline 8 & else & \\
\hline 9 & $A[i d x][++$ tail $] \leftarrow w ;$ & \\
\hline 10 & new_path $\leftarrow$ TRUE & \\
\hline 11 & depthFirst (w); & \\
\hline
\end{tabular}

As an example, Table 1 lists the independent paths discovered by a call of depthFirst $\left(s_{1}\right)$ on the MST spanning structure given in Figure 3. The choice of which unvisited edge to traverse first in line 2 is arbitrary. In the example listed in Table 1, the edge $\left\langle s_{1}, d_{2}\right\rangle$ is explored first resulting in a call to depthFirst $\left(d_{2}\right)$. Since there are no outgoing untraversed edges from $d_{2}$, execution returns and resumes at depthFirst $\left(s_{1}\right)$. A new independent path is created starting with the exploration of 
edge $\left\langle s_{1}, d_{3}\right\rangle$ and a commensurate call to depthFirst $\left(d_{3}\right)$. Notice that the last vertex in each independent path has degree one and consequently is a singleton if it is a demand vertex.

Table 1. Six independent paths for the MST in

Figure 3 derived by depthFirst called with the supply vertex $s_{1}$.

\begin{tabular}{|l|l|}
\hline & Independent Paths in $\boldsymbol{A}$ \\
\hline 1 & $s_{1}, d_{2}$ \\
\hline 2 & $s_{1}, d_{3}, d_{1}$ \\
\hline 3 & $d_{3}, s_{2}, d_{4}$ \\
\hline 4 & $s_{2}, d_{6}, d_{9}, s_{4}, d_{11}, d_{7}, s_{3}$ \\
\hline 5 & $d_{7}, d_{5}$ \\
\hline 6 & $d_{9}, d_{8}, d_{10}$ \\
\hline
\end{tabular}

The time complexity of the depthFirst routine is simple to compute. The routine visits each of the $\mathrm{O}(|V|)$ edges in the spanning structure once and does a constant amount of work at each. Hence the running time of the depthFirst routine is $\mathrm{O}(|V|)$.

To understand the behavior of getRobustSet it is necessary to understand the context in which it is called. As previously described the MST is augmented with edges until all the vertices are robust. After each edge is added to a singleton, getRobustSet is called and returns the new set of robust vertices.

Listing 2. The get RobustSet routine is called after every single edge insertion.

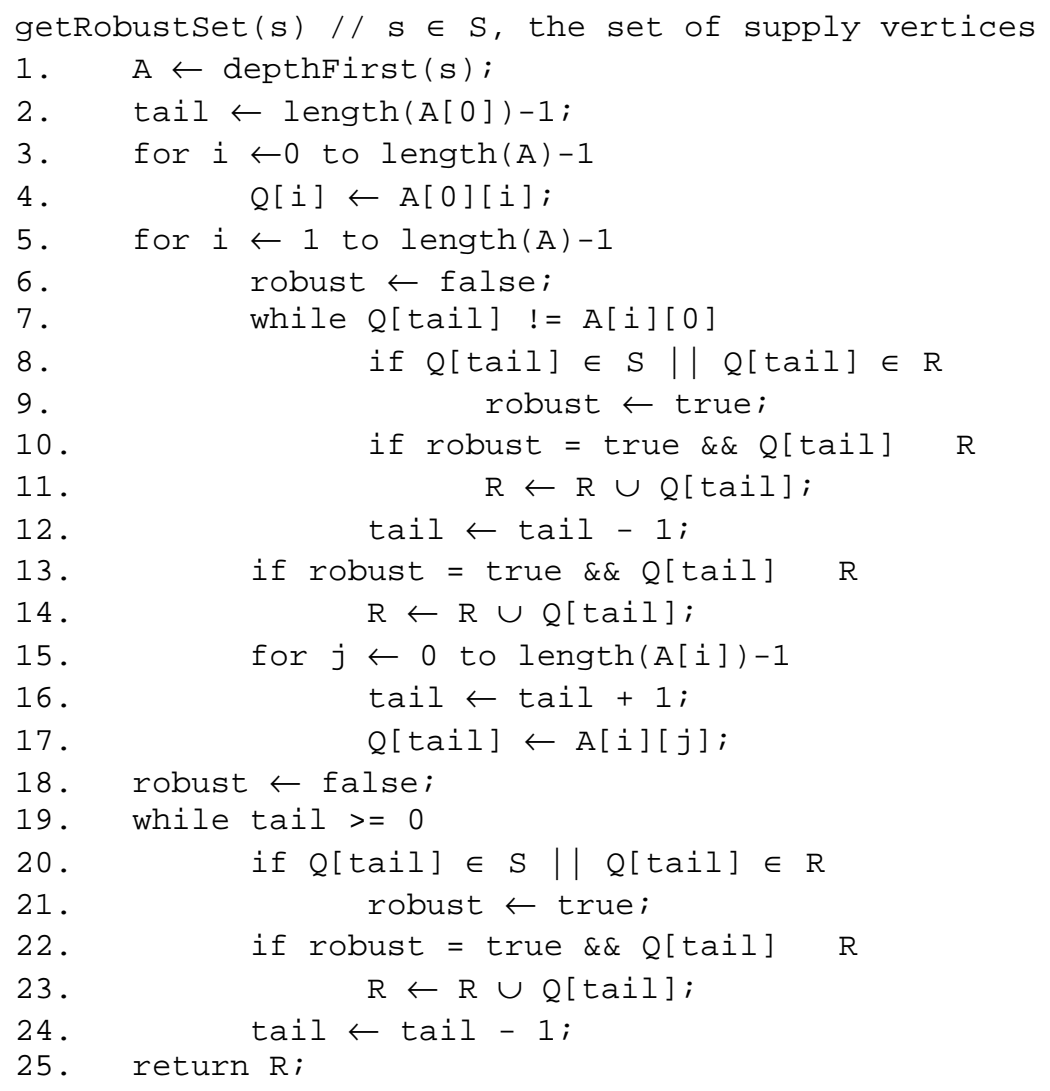


As seen in Listing 2, getRobustSet calls depthFirst, with an arbitrary supply vertex $s$. After the depthFirst determines the set of independent paths in the spanning structure, the getRobust routine will utilize these independent paths to construct paths, all rooted at $s$, through the spanning structure.

The current path through the spanning structure is maintained in an array $Q$. The first element of $Q$ will always be $s$, a supply vertex used in the call to depthFirst. The getRobustSet routine initializes the path of vertices, $Q$, to the first independent path discovered by the depthFirst routine (lines 3-4). Next, getRobustSet backward scans the $Q$ array from the last element towards the first element, and looks for a robust vertex, stopping only when it finds the first element of the next independent path in $\boldsymbol{A}$ (line 7). Since the path implied by $Q$ is always rooted at a supply vertex, any robust vertex encountered will, by Lemma 2 , result in all of the vertices between the robust vertex and $s$ being classified as robust. However, this conversion will not happen all at once in the getRobustSet routine. When a robust vertex is encountered (lines 8) the robust flag is set (line 9) and the vertices in $Q$, up to the first vertex of the next independent path in $A$, are then moved into $R$ (lines 10,11 and 13,14). The next independent path is then appended to $Q$ (lines 15-17). After the last independent path in $\boldsymbol{A}$ has been appended, the backward scan of $Q$ is continued all the way back to $s$, the argument of getRobustSet (lines 19-24).

To better understand the getRobustSet procedure, its execution is presented using the independent paths from Table 1. Table 2 shows $Q$ for each referenced independent path, labeled "IP". The column labeled "Backscan" contains the first vertex of the next independent path and consequently the end of the backscan of $Q$ (line 7). The column labeled " $R$ " lists the vertices added to the robust set $\boldsymbol{R}$ in lines 11, 14, or 23 of the getRobustSet algorithm.

Table 2. The evolution of the data structures in $\operatorname{getRobustSet}\left(s_{1}\right)$ when called with the MST in Figure 3.

\begin{tabular}{|l|l|l|l|}
\hline IP & \multicolumn{1}{|c|}{$Q$} & \multicolumn{1}{|c|}{ Backscan } & \multicolumn{1}{c|}{$R$} \\
\hline 1 & $s_{1}, d_{2}$ & $s_{1}$ & $\left\{s_{1}\right\}$ \\
\hline 2 & $s_{1}, d_{3}, d_{1}$ & $d_{3}$ & $\left\{s_{1}\right\}$ \\
\hline 3 & $s_{1}, d_{3}, s_{2}, d_{4}$ & $s_{2}$ & $\left\{s_{1}, s_{2}\right\}$ \\
\hline 4 & $s_{1}, d_{3}, s_{2}, d_{6}, d_{9}$, & $d_{7}$ & $\left\{s_{1}, s_{2}, s_{3}, d_{7}\right\}$ \\
& $s_{4}, d_{11}, d_{7}, s_{3}$ & & \\
\hline 5 & $s_{1}, d_{3}, s_{2}, d_{6}, d_{9}$, & $d_{9}$ & $\left\{s_{1}, s_{2}, s_{3}, d_{7}\right.$, \\
& $s_{4}, d_{11}, d_{7}, d_{5}$ & & $\left.d_{11}, s_{4}, d_{9}\right\}$ \\
\hline 6 & $s_{1}, d_{3}, s_{2}, d_{6}, d_{9}$, & $s_{1}$ & $\left\{s_{1}, s_{2}, s_{3}, d_{7}\right.$, \\
& $d_{8}, d_{10}$ & & $d_{11}, s_{4}, d_{9}, d_{6}$, \\
& & & $\left.d_{3}\right\}$ \\
\hline
\end{tabular}

Showing the correctness of the getRobustSet algorithm proceeds in two steps, showing that it returns a correct robust set when called with an MST and that it correctly augments the set of robust vertices under a single edge insertion operation (see Figure 4 for the augmented spanning structure). In both cases it is necessary to show that the classification of a vertex as robust cannot affect the classification of a vertex on an indepdent path of $\boldsymbol{A}$ which has already been processed.

The first invocation of getRobustSet is run on an MST of the network where all paths end in singletons. Thus it is not possible for a vertex which is discovered at some point to be robust to affect a vertex in another independent path because there are no cycles in a MST and consequently no connections between the branches of a tree.

To show that getRobustSet correctly augments the set of robust vertices under a single edge insertion operation, assume that all preceding invocations of getRobustSet have correctly 
identified the set of robust vertices. An edge is added to the spanning structure from a singleton vertex, $v$, to a redundant vertex, $w$. After the edge, $e=\langle v, w\rangle$, is added to the spanning structure, getRedundentSet is called to augment the robust set. The edge $\langle v, w\rangle$ will cause the vertices on the path from $s$ to $v$ to become robust. No other vertices in the spanning structure are affected because $w$ is already robust and there is at most one path from $v$ to $s$ (that's why $v$ is a singleton).

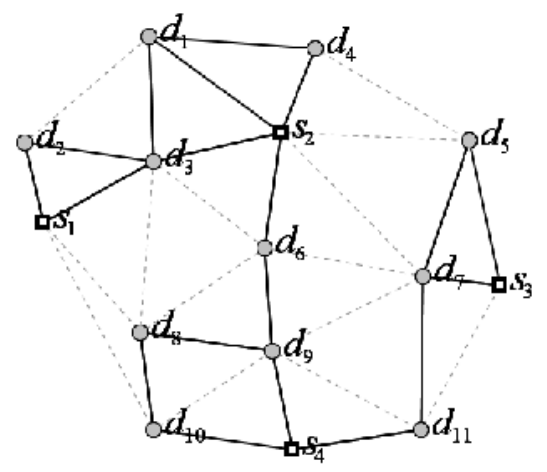

Figure 4. The spanning structure resulting from the edge augmentation of the MST in Figure 3.

There are times when the set of edges provided by the Delaunay triangulation proves to be insufficient for the augmentation process to find an edge which increases the size of the robust vertices as shown in Figure 5. In such cases the augmentation algorithm looks for the shortest edge which connects the singleton to a supply vertex.
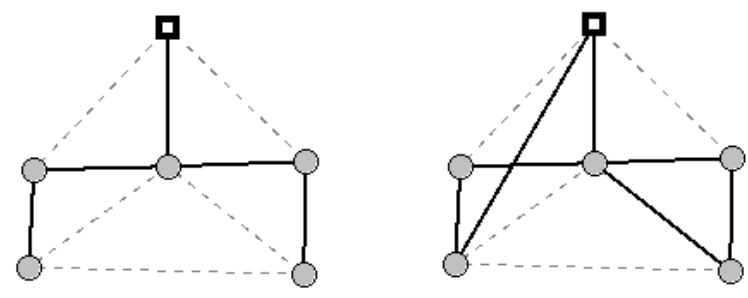

Figure 5. Example of a 2-ESD connectivity which uses an edge outside of the Delaunay triangulation.

The augmentation process ends when the set of robust vertices is equal to the set of supply and demand vertices. At this point the graph is 2-ESD connected. The second phase of the algorithms then takes over, reducing the cost of the 2-ESD connected network while maintaining the redundancy of the network.

\subsection{Edge Reduction/Refactoring}

The graph resulting from the augmentation of the MST is 2-ESD connected, but may not be optimal in terms of cost. The cost of the spanning structure can be reduced by eliminating unnecessary edges using reduction heuristics. The removal of an edge may have two negative consequences, eliminating the robustness of a vertex or breaking the graph into two components. In order to ensure that the application of a reduction heuristic does not create an illegal configuration, the edge replacement is applied only if getRobustSet returns a complete set of vertices under a tentative application of the edge reduction. The three reduction heuristics utilized are greedy-based and often yield a solution that is close to optimal. 


\subsubsection{Triangle Inequality}

The first reduction heuristic looks to replace a pair of edges with a single edge based on the triangle inequality. A 2-ESD redundant network containing a supply vertex with degree $3+$ or a demand vertex with degree $4+$ can have its cost reduced by replacing the two most expensive edges $\left\langle v, v_{1}\right\rangle$ and $\left\langle v, v_{2}\right\rangle$ with the edge $\left\langle v_{1}, v_{2}\right\rangle$ where $v$ is the high degree vertex. This replacement always reduces the cost of the network because the triangle inequality states that the sum of any two edges of a triangle is always greater than the length of the third edge [12].

Although the replacement of edges does not affect the robustness of the vertices, it can split the graph into two components. Thus, each step in the application of this reduction heuristic must be followed by a check for 2-ESD. First, the new edge is added to the spanning structure. Then each expensive edge is removed from the spanning structure (one at a time), getRobustSet is called, and the returned set of robust vertices, $\boldsymbol{R}$, is checked. If $\boldsymbol{R}$ is changed, the replacement is rejected and the original state is reinstated, otherwise the substitution is retained. Figure 6 shows the application of this first reduction heuristic.

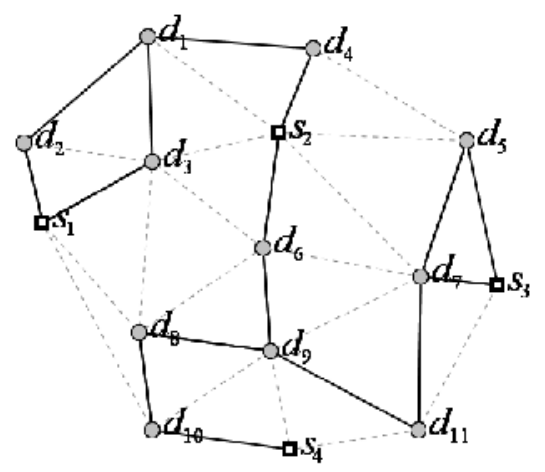

Figure 6. The application of the triangle inequality to replace edges $\left\langle d_{2}, d_{3}\right\rangle$ and $\left\langle d_{1}, d_{3}\right\rangle$ with $\left\langle d_{1}, d_{2}\right\rangle$ and then replace edges $\left\langle d_{1}, s_{2}\right\rangle$ and $\left\langle d_{3}, s_{2}\right\rangle$ with $\left\langle d_{1}, d_{3}\right\rangle$.

\subsubsection{Edge Removal}

The second reduction heuristic looks for edges which can be removed. Instead of attempting to eliminate each of the edges in the 2-ESD network one at a time, candidate edges for removal are identified by the degree and type of their terminals. Let $s$ be a supply vertex and $d$ be a demand vertex. The following three rules specify the conditions sufficient to remove an edge.

Remove edge $\langle s, d\rangle$ when $\operatorname{deg}(s)>1$ and $\operatorname{deg}(d)>2$

Remove edge $\left\langle s_{1}, s_{2}\right\rangle$ when $\operatorname{deg}\left(s_{1}\right)>1$ and $\operatorname{deg}\left(s_{2}\right)>1$

Remove edge $\left\langle d_{1}, d_{2}>\right.$ when $\operatorname{deg}\left(d_{1}\right)>2$ and $\operatorname{deg}\left(d_{2}\right)>2$

For example, the first rule states that an edge whose one terminal is a supply vertex with degree $1+$ and the other is a demand vertex with degree 2+ may be removed. It can be shown that this removal will not affect the robustness of the vertices, but may affect the connectedness of the graph. Thus, this optimization step is applied with the condition that getRobustSet returns a complete set of vertices after the edge has been removed. The application of the second reduction heuristic is shown in Figure 7. 


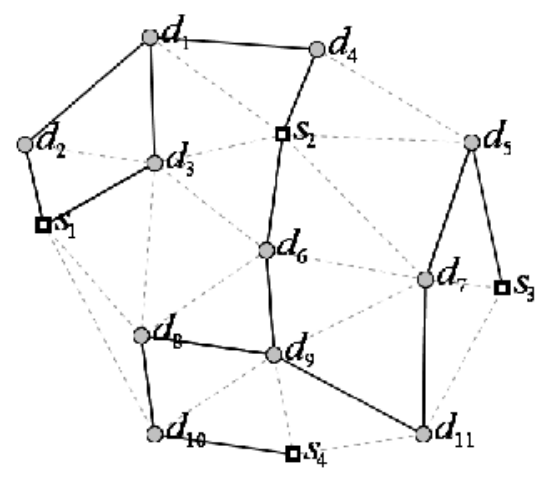

Figure 7. The application of the reduction heuristic in the removal of edge $\left\langle d_{7}, s_{3}\right\rangle$.

\subsubsection{Edge Refactoring}

The previous reduction heuristic looks for an edge which terminates in high degree vertices. The edge refactoring reduction heuristic looks for a path terminating in high degree vertices. One edge incident to each of the high degree vertices may be removed and replaced with an edge joining the terminating vertices whose degree have also been reduced. For example, in Figure 7 vertices $s_{1}$ and $d_{1}$ are high degree, based on the first rule in section $C 2$. Edges $\left\langle s_{1}, d_{3}\right\rangle$ and $\left\langle d_{1}\right.$, $\left.d_{2}\right\rangle$ are removed and replaced with a cost-reducing edge $\left\langle d_{2}, d_{3}\right\rangle$. This exchange results in the 2ESD connected network shown in Figure 8. In this case, vertices $d_{2}$ and $d_{3}$ are terminating vertices. This optimization step is also applied with the condition that getRobustSet returns a complete set of vertices after refactoring has occurred.

This reduction heuristic is computationally intensive because both the number of paths and the set of candidate replacement edges are quite large. The set of candidate replacement edges is large because it includes edges not in the Delaunay triangulation, similar to the situation shown in Figure 5 .

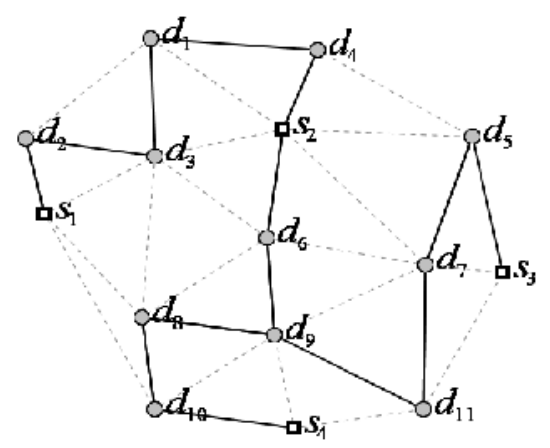

Figure 8. The application of the refactoring heuristic to replace edges $\left\langle s_{1}, d_{3}\right\rangle$ and $\left\langle d_{1}, d_{2}\right\rangle$ with $\left\langle d_{2}, d_{3}\right\rangle$.

\section{EXPERIMENTS}

The proposed algorithm was implemented in Java v1.6 and tested on a PC with Windows 7 32-bit Enterprise OS. The computer has 4 GB RAM and the CPU is Intel Core 2 Quad Q8200 @ $2.33 \mathrm{GHz}$. Although the greedy approach may not yield an optimal solution, experiments are conducted to compare the cost for networks with and without fault tolerance. Cost is taken to have two meanings: the cost associated with the Euclidean distance to form a network, and the cost associated with the network's computation time. 
To address different network complexities, the number of vertices in the network covers 10,50 , 100 and then increments by 100 up to 800 vertices. Coordinates are randomly generated within a $600 \times 1000$ rectangle. The density of demand vertices, with respect to the number of vertices, is varied between $10 \%$ and $90 \%$. Note that a density of $0 \%$ implies that all the vertices are supply vertices. Consequently the connection requirements of the supply vertices imply that the 2-ESD network is simply an MST.

Figure 9 depicts the Euclidean distance cost associated with adding redundancy to a MST. The vertical axis is the percentage increase in the cost of the redundant network over the MST. The data is collected by averaging the percentage increase in cost over 20 randomly generated networks. The increase in cost is plotted against the number of vertices in the network and the density of demand vertices.

The large percentage increase in cost associated with adding redundancy to small networks is a side effect of their small size. The addition of a single edge to a small network having just a few edges represents a significant portion of the network cost. More representative behavior is exhibited in larger networks. Increasing the proportion of demand vertices results in a larger increase in the cost because each demand vertex must have two independent paths to a supply vertex, whereas a supply vertex may have degree one if it's connected to another supply vertex.

The computation time required to add redundancy to a network increases as the number of vertices increase as shown in Figure 10. However, for larger networks the minimum computation time occurs when there are an equal number of demand and supply vertices.

To explain this saddle point, Figure 10 shows the running time of each of the 5 phases of the algorithm (MST construction, edge augmentation (Section III.B), Triangle Inequality (Section III.C1), Edge reduction (Section III.C2), and Edge refactoring (Section III.C3)) are plotted for a network with 800 vertices and having a demand vertex density between $10 \%$ and $90 \%$. When the density of demand vertices is low, the large number of supply vertices requires a lot of time to be spent on refactoring, in an attempt to reduce the degree of the supply vertices. A high density of demand vertices implies a proportionally large number of singleton vertices. Thus, a lot of time is spent on augmenting the network to make it redundant.

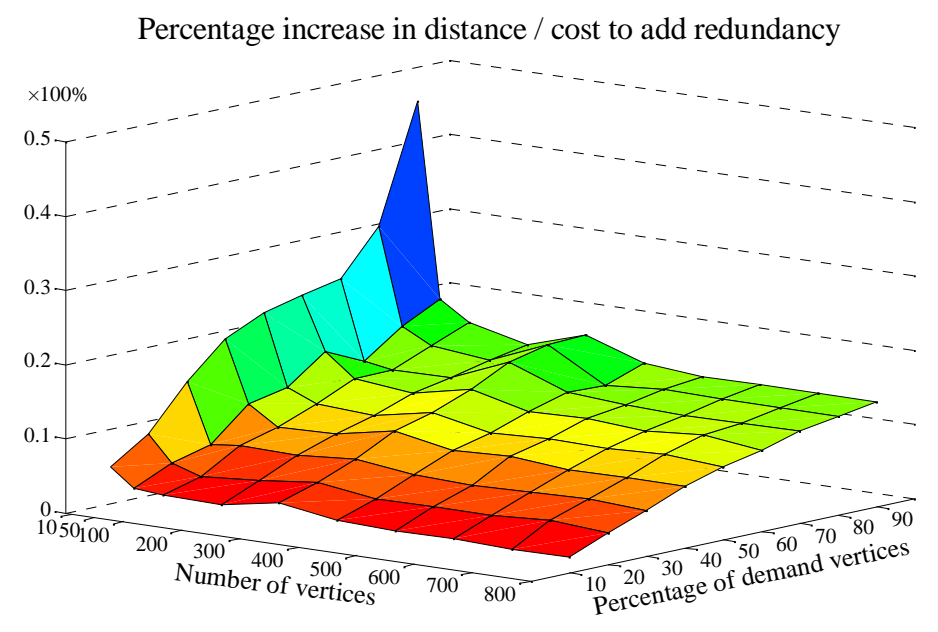

Figure 9. The cost of adding redundancy to a MST expressed as a percentage of the MST's cost. 


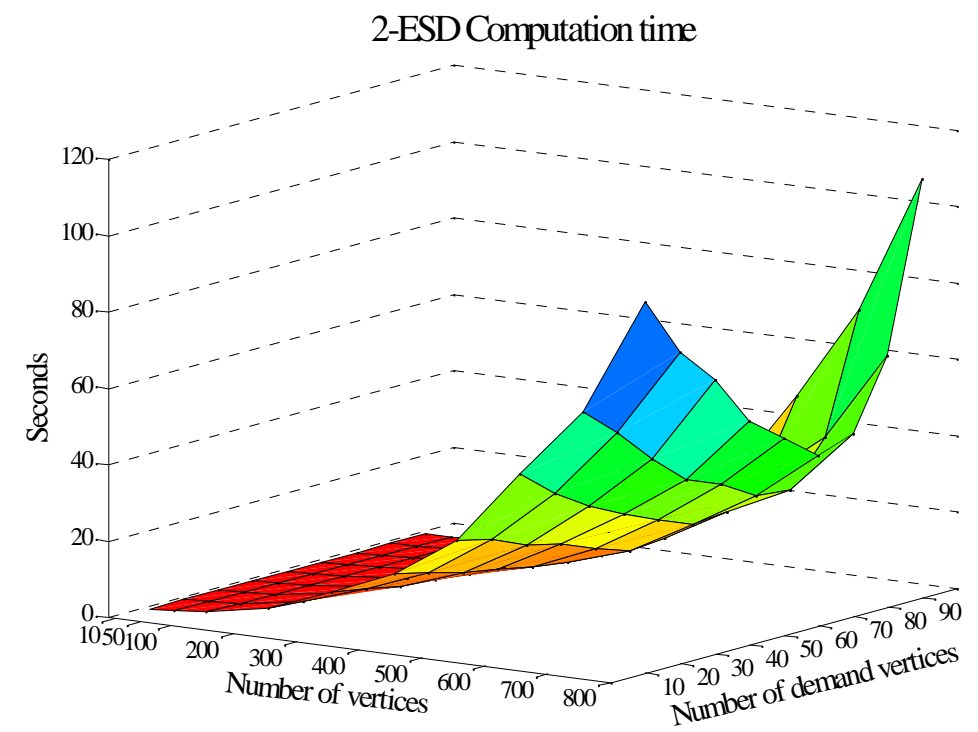

Figure 10. Computation time cost associated with adding redundancy to a network.

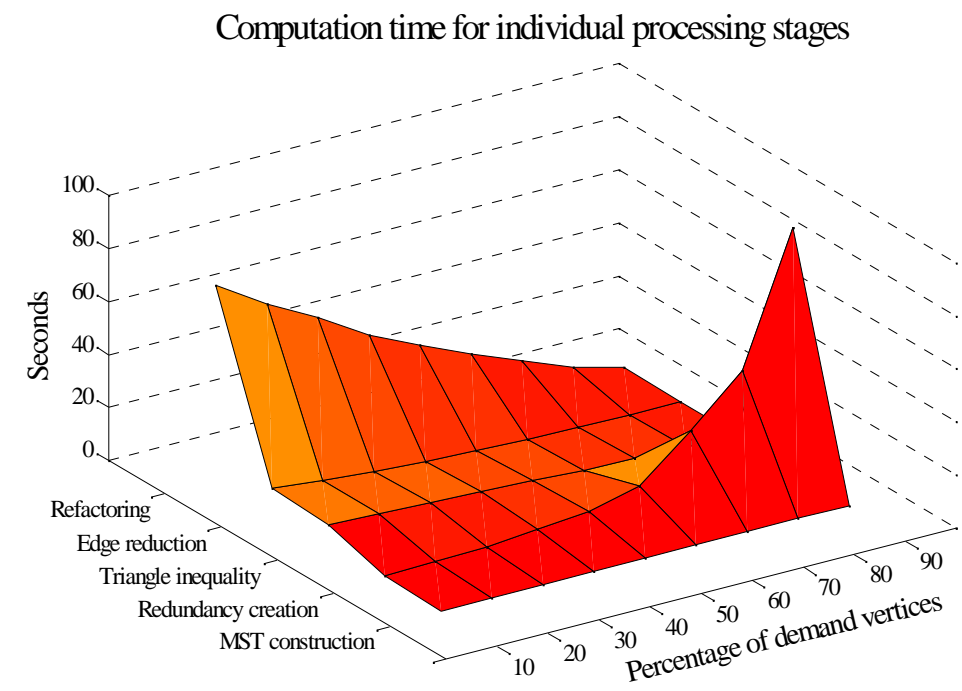

Figure 11. Computation time cost for individual stages in the 2-ESD development process.

\section{CONCLUSIONS AND FUTURE WORK}

A new class of NP-hard problem, the 2-ESD connected network, is introduced. This problem seeks to establish connectivity between two types of functionally distinct entities, namely, one which supplies services to fulfill demands of the other. The demand entities must be able to tolerate a connection break-down in the infrastructure in order to stay served. This problem has applications to areas, such as power systems, supply chain and logistics.

The proposed algorithm adopts a two-phase approach to solve this problem. After constructing an MST to connect all the entities, the first phase generates redundant connections for the demand entities to meet their fault tolerance requirement. The second phase incorporates a series of 
greedy procedures to refactor and eliminate unnecessary connections to decrease the cost of the entire infrastructure. Experimental results show that adding redundancy may increase the cost of the infrastructure by up to $20 \%$ for large network.

Future work in this area includes looking at more effective optimization heuristics while decreasing the running time of the algorithm. Since the luxury of designing a new network topology from scratch is rare, there is a need to examine how to augment an existing nonredundant network to make it redundant. A related problem is how to add supply and demand vertices and edges to an existing network so that the connection requirements of a 2-ESD network are satisfied.

This work considers space to have a uniform cost. In some applications this may not be the case, for example when installing transmission lines the cost to traverse an open plain may be greater than when traversing a mountainous regions. Further work needs to be directed at this practical variation.

\section{REFERENCES}

[1] Brown, R., Electric Power Distribution Reliability, 2nd Ed., CRC Press, 2009.

[2] Pourbeik, P.; Kundur, P.S.; and Taylor, C.W.; , "The anatomy of a power grid blackout - Root causes and dynamics of recent major blackouts," Power and Energy Magazine, IEEE, vol.4, no.5, pp.22-29, Sept.-Oct. 2006.

[3] ERCOT System Planning Study, "Competitive Renewable Energy Zones (CREZ) Transmission Optimization Study,” April 2, 2008.

[4] Wada, K.; Luo, Y.; and Kawaguchi, K., "Optimal Fault-Tolerant Routings for Connected Graphs," Information Processing Letters, Vol. 41, Iss. 3, Mar. 1992, pp. 169 - 174.

[5] Liu, Y. L.; and Wang, Y-L.; Guan, D.J.; "An optimal fault-tolerant routing algorithm for double-loop networks," Computers, IEEE Transactions on, vol.50, no.5, pp.500-505, May 2001.

[6] Moraes, R.E.N.; Ribeiro, C.C.; Duhamel, C.; , "Optimal solutions for fault-tolerant topology control in wireless ad hoc networks," Wireless Communications, IEEE Transactions on , vol.8, no.12, pp.5970-5981, December 2009.

[7] Cheng, L.; Carter, J.; and Dai, D., "An Adaptive Cache Coherence Protocol Optimized for ProducerConsumer Sharing", IEEE 13th International Symposium on High Performance Computer Architecture, Scottsdale, AZ, pp. 328 - 339, February 2007.

[8] Hou, P.; and Hsu, Y., "Finding Dominant Links of Emergency Network with Respect to Earthquake Disaster," Journal of the Eastern Asia Society for Transportation Studies, Vol. 6, pp. 77 - 90, 2005.

[9] Papadimitriou C.; and Steiglitz K., Combinatorial Optimization Algorithms and Complexity. Mineola, New York: Dover Publications, 1998.

[10] A Computational Investigation of Heuristic Algorithms for 2-edge-Connectivity Augmentation, Jørgen Bang-Jensen, Marco Chiarandini *, Peter Morling, Networks, Volume 55 Issue 4, Pages 299 325.

[11] Garey, M.R.; and Johnson D.S. (1979). Computers and Intractability: A Guide to the Theory of NPCompleteness. W.H. Freeman.

[12] O’Rourke, J., Computational Geometry in C, Cambridge University Press, 2000.

[13] Berg, M.D.; Cheong, O.; Kreveld, M.V., Overmars M., Computational Geometry: Algorithms and Applications, Springer, Nov. 19, 2010. 


\section{AUTHORS}

Dr. Wang received his Ph.D. in Computer Science from State University of New York at Albany in 2002. He is currently an Associate Professor in Computer Science and Software Engineering department at Penn State Erie, The Behrend College. His research interests are in software design, software architecture, software reliability, pattern recognition, machine learning, adaptive systems, and game AIs.

Dr. Chris Coulston received his B.A. in Physics in 1989 from Slippery Rock University, his B.S. 1991, the M.S. 1994 and the Ph.D. 1999 in Computer Engineering, all from the Pennsylvania State University. Dr. Coulston taught at the University Park campus from 1993-1998. He is chair of the Computer Science and Software Engineering Department beginning January 2012. He was the former chair of Electrical, Computer, and Software Engineering programs. He received the best paper award for Constructing Exact Ooctagonal Steiner Minimal Trees, at the Great Lakes Symposium on Circuits and

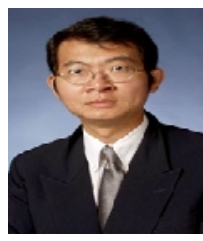
Systems, April 2003 and co-authored Design for Electrical and Computer Engineers: Theory, Concepts and Practice. New York: McGraw-Hill Higher Education, 2005, 300 pp., ISBN 0-07-319599-5 with Dr. Ralph Ford, director of the School of Engineering.

Dr. Robert Weissbach is currently an associate professor of engineering at Penn State Erie, The Behrend College. From October 2007 through June 2008, he was a visiting researcher at Aalborg University in Aalborg, Denmark. His research interests are in renewable energy, energy storage, power electronics and power systems.

Dr. Tang received her Ph.D. in Computer Science from SUNY Albany in 2003. She is currently an Associate Professor in Computer and Information Science department at Gannon University. Her research interests are in software design, software metrics, software architecture, software reliability, software testing, and swarm intellige

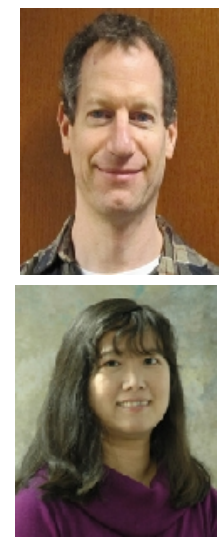

